\title{
Automatic Handling Device for Disc Cover Parts
}

\author{
GuangZhen Cheng ${ }^{1,}$, , ShiYang Wei ${ }^{1, b}$, Qian Zhou ${ }^{1, c}$ \\ ${ }^{1}$ Huzhou Teachers College, Huzhou, 313000, P.R. China \\ achgzh169@126.com, ${ }^{b} 1396811300 @ q q . c o m,{ }^{c} 1151221032 @ q q . c o m$
}

Keywords: Handling device; Disc Cover Parts; Automatic;

Abstract. This paper introduced an automatic handling system. It composed of mechanical structure, electrical control system. The mechanical structure including frame body, moving car, hydraulic system. It can achieve horizontal moving, up and down moving, clamping, loosen. According to the working requirements, handling device can be manual control, also can automatic control, complete parts handling. It can adapt to bad working conditions, can work continuously. Thereby improve working conditions, reduce labor intensity and improve work efficiency.

\section{Introduction}

To design a set of work piece automatic feeding mechanism, with stepping conveyor for processing plate set of transport to the front of the machine tool, parts artificial press the start button, feeding mechanism from the initial position, complete fall, clamping, rising, moves to the right, down, loosen, up, left, back to the initial position to stop, to implement a work cycle. Control of stepping conveyor, automatic feeding mechanism, machine tool working rhythm is consistent, can be repeated, according to requirements of the work cycle until the press the stop button automatically. For convenient installation, debugging, maintenance, the control system have five kinds of work way, it is the origin, manual, automatic, single step and single cycle.

\section{Structural Composition}

The structure is shown in Fig1, which is composed of a frame body, a moving device, a horizontal moving oil cylinder YG1, an upper and a lower movable oil cylinder YG2, a clamping oil cylinder YG3, a conveyor, a working table and a stroke switch. The oil cylinder YG1 is fixed on the frame body, and the oil cylinder YG1 piston rod is fixedly connected with the mobile device, which drives the moving device to realize horizontal movement along the guide rail of the mobile device, and the upper and lower moving oil cylinder YG2 is fixed on the moving device. The oil cylinder YG2 piston rod is fixedly connected with the clamping oil cylinder YG3, the oil cylinder YG2 piston rod can be moved up and down to move up and down, and the clamping oil cylinder YG3 drives the clamping block to realize the clamping and loosening. When the automatic mode of operation, rely on the travel switch to achieve the action conversion. Travel switch installation location accurate, handling device motion position accurately. This design were installed upper limit position switch SQ0, down limit switch SQ1, left limit travel switch SQ2, right limit travel switch SQ3. The limit switch SQ0, SQ1 is relatively fixed with mobile device. The limit switch SQ2, SQ3 is relatively fixed with frame. The parts detecting switch SQ4 is fixed on the frame body, to ensure that there is no object on the working table, only then the feeding mechanism is allowed to drop and the parts is put into the working table.

\section{Hydraulic and Electrical Control System}

The hydraulic system of the automatic feeding mechanism of the disc cover parts is shown in Fig2. Executive agencies including, left and right moving cylinder YG1. Up and down cylinder YG2, loosen and clamping cylinder YG3, there are double piston with the cylinder YG3, intermediate into the oil chamber, left and right cavity of the oil return, left piston to move to the left, right piston shifted to the right, release the parts, when the left and right cavity into the oil chamber, intermediate cavity the oil back, left piston shifted to the right, the right piston to move to the left, 
clamping parts. Three implementation oil cylinders YG1, YG2, YG3 was controlled by the four position three way reversing valve YV1, four position three way reversing valve YV2, two position four way reversing valve YV3, To achieve left and right moving, up and down moving, the clamping and loosening.

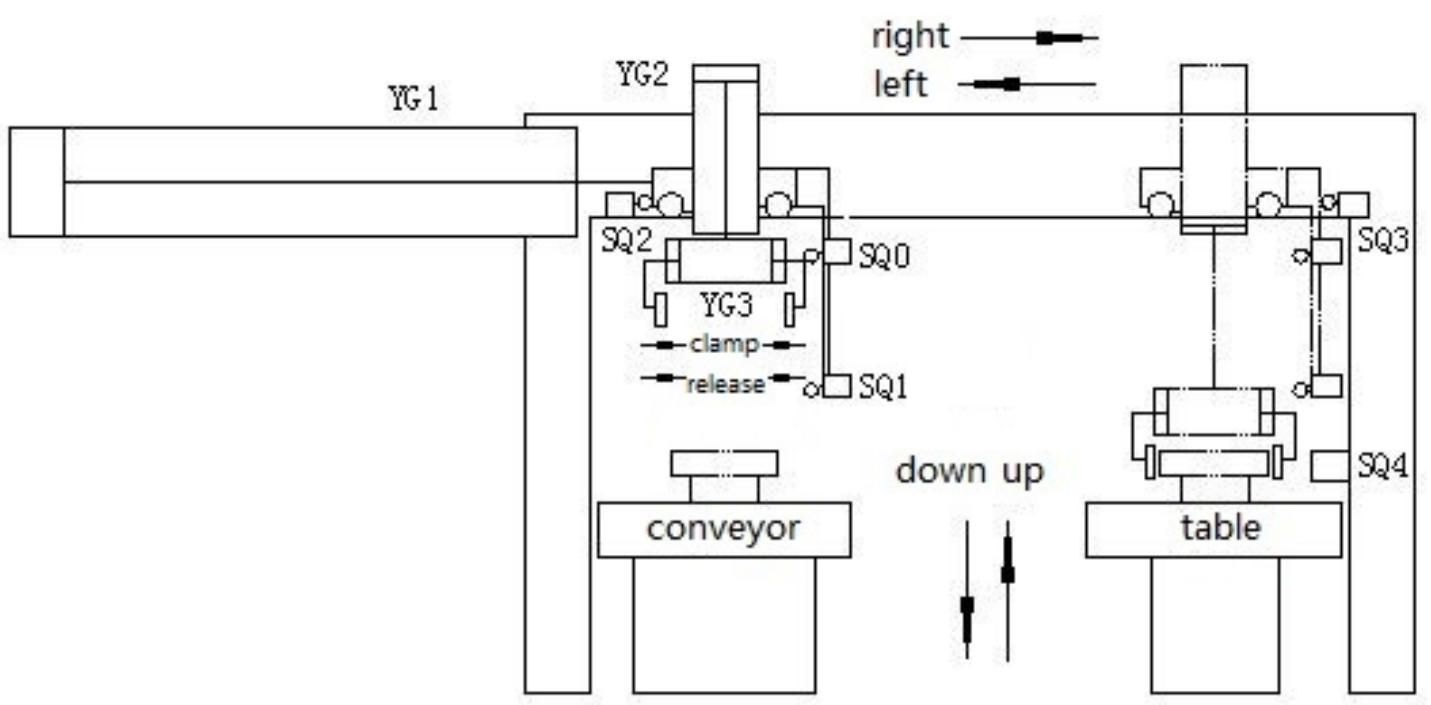

Fig. 1 Structure schematic diagram

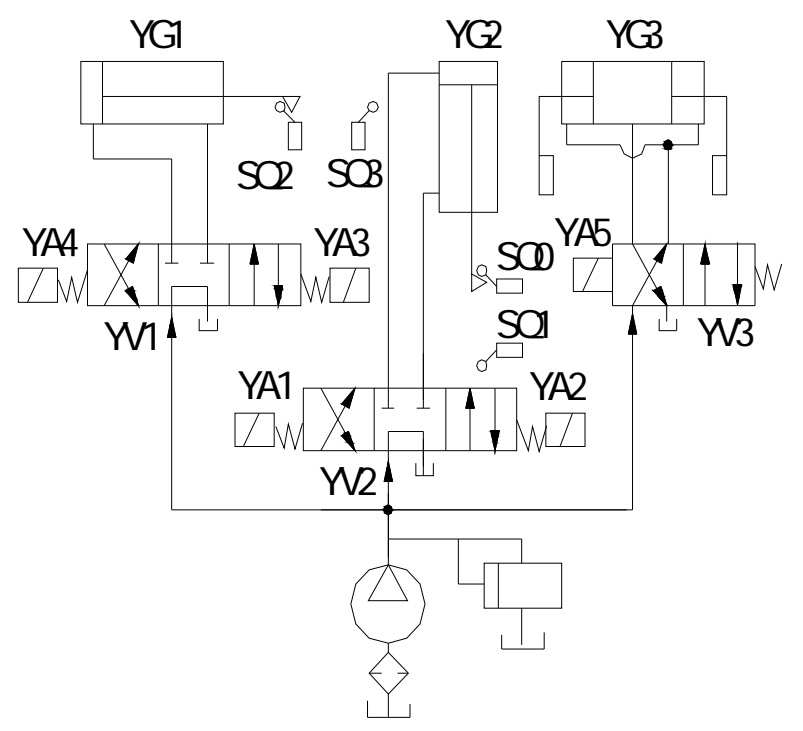

Fig. 2 Hydraulic system diagram

Electrical control system used PLC system. for the selection of PLC, mainly consider the scanning speed, memory capacity, function module, instruction article number, number of input, output, should also consider its economic, practical and working environment. The final selection was FX2N - 48 MR. PLC input address allocation and element function as shown in table 1. PLC output address allocation and element function as shown in table 2. 
Table 1 PLC input address

\begin{tabular}{lcc}
\hline Functional & Device & Input \\
\hline Manual mode & SA-1 & X010 \\
Back to the origin & SA-2 & X011 \\
Single-step mode & SA-3 & X012 \\
Single-cycle mode & SA-4 & X013 \\
Automatic mode & SA-5 & X014 \\
Back to origin & SB5 & X015 \\
Auto start & SB6 & X016 \\
Auto stop & SB7 & X017 \\
Up limit position & SQ0 & X000 \\
Down limit position & SQ1 & X001 \\
Left limit position & SQ2 & X002 \\
Right limit position & SQ3 & X003 \\
Manual rise & SB1 & X004 \\
Manually drop & SB2 & X005 \\
Manual to right & SB3 & X006 \\
Manual to left & SB4 & X007 \\
Manual clamping & SB8 & X020 \\
Manual release & SB9 & X021 \\
Part inspection & SQ4 & X022 \\
\hline
\end{tabular}

Table 2 PLC output address

\begin{tabular}{lcc}
\hline Functional & Device & Output \\
\hline Rise electromagnet & YA1 & Y000 \\
Down electromagnet & YA2 & Y001 \\
Right electromagnet & YA3 & Y002 \\
Left electromagnet & YA4 & Y003 \\
Clamp electromagnet & YA5 & Y004 \\
Original light & EL & Y005 \\
\hline
\end{tabular}

\section{Work Process Analysis}

When turned on, the knob switch turned to the original position SA2, press the homing button SB5, institutions back into the origin, the feeding mechanism is in the leftmost, uppermost. All $\mathrm{s}$ electromagnet YA1, YA2, YA3, YA4, YA5 power off, this state was called in situ. Then the rotary switch turn to the appropriate operating mode, you can start work, the main actions are, the working bodies rise and down, left and right. Clamping device clamp and loosen. When the manual mode, press the corresponding manual button, complete the appropriate action. When the automatic operation mode, press the start button SB6, institutions in accordance with a predetermined program, start automatic work cycle. Automatically work process as follows.

Downward movement and clamping. Select the Auto mode, push the start button SB6, the electromagnet YA2 power on, the solenoid YV2 in the right position, For the cylinder YG2 oil go into the upper chamber and the under chamber return, the piston down until press the limit switch SQ1, electromagnet YA2 power off, down moment to stop, while the energized electromagnet YA5 power on, the solenoid YV3 in the left position, For the cylinder YG3 the oil go into the left and right chamber, the intermediate chamber back to the oil, the piston moves to clamp the parts.

Rise and move to the right. After clamping the parts, the electromagnet YA1 power on, the solenoid YV2 in left position, the cylinder YG2 lower chamber into oil and upper chamber return oil, the piston moves until the pressed limit switch SQ0, the electromagnet YA1 power off, stop rising, while the electromagnet YA3 power on, the solenoid YV1 in the right position, the cylinder 
YG1 left chamber into oil and right chamber return oil, the piston move to right, until the pressed limit switch SQ3, the electromagnet YA3 power off, right moment stopped.

Downward movement and loosen. Stop right at the same time, the electromagnet YA2 power on, the solenoid YV2 was at right position, the cylinder YG2 upper chamber into oil and the lower chamber back to oil, the piston move down until press the limit switch SQ1, the electromagnet YA2 power off, down moment stop, while the electromagnet YA5 power off, the solenoid YV3 was at right position, the cylinder YG3 intermediate go into oil, left and right chamber return oil, the piston moves, the parts was released.

Up movement and move to the left. After the released the parts, the electromagnet YA1 power on, the solenoid YV2 was at left position, the cylinder YG2 lower chamber into oil and upper chamber return oil, the piston up until pressed limit switch SQ0, the electromagnet YA1 power off, the piston stopped rising, while the electromagnet YA4 power on, the solenoid YV1 was at left position, the cylinder YG1 right chamber into oil and left chamber back to oil, the piston moved left until the pressed limit switch SQ2, the electromagnet YA4 power off, the piston stopped left, went back into the origin, completed a cycle of work.

Next, the electromagnet YA2 power on, the solenoid YV2 was at right position, the cylinder YG2 upper chamber into oil and under chamber return oil, the piston moved down, the next work cycle started.

\section{Conclusion}

1) Run the PLC program control the working bodies, and were developed manually back into place, single-step, single cycle, continuous work and so are several different ways to suit different operational needs for easy installation, equipment maintenance.

2) Program is provided with a protective circuit, interlocking relationship, to ensure safe and reliable system operation.

3) Hydraulic system three-way valve, the median function using $M$ functions, in favor of energy-saving hydraulic pump unloading.

4) In order to ensure that no artifacts on the table to drop, the parts detection switch installed in the frame body SQ4, there is no guarantee that when the object table before allowing the parts feeding mechanism fell into the table, and avoid working bodies workbench collision.

\section{References}

[1] S.H. Chen: Machinery equipment electrical control (South China University pres, China 2002).

[2] Y.S. Fan: Electrical Control and PLC applications (China Electric Power Press, China 2006) (in Chinese).

[3] Y.J. Jia, D.Y. Zhang, G.Z. Cheng: Applied Mechanics and Materials Vol. 164 (2012) pp 344-347.

[4]G.Z.Cheng:Automatic grinding machine electrical control system[J] Manufacturing Technology \& Machine, 2011 (9): 81-83.

[5] Z.Q. Qi. Machine Electrical Control Technology(Machinery Industry Press, China 2002).

[6] Y.J. Jia and G.Z. Cheng: Hydraulic and pneumatic, Sep.2009, pp4-6 (in Chinese). 